\title{
Tentorial Venous Anatomy: Variation in the Healthy Population
}

\author{
(D).S. Rosenblum, (D).M. Tunacao, (D) V. Chandrashekhar, (D)A. Jha, (D) M. Neto, (DC. Weiss, (D). Smirniotopoulos,
}

(D) B.R. Rosenblum, and (D).D. Heiss

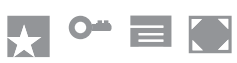

\begin{abstract}
BACKGROUND AND PURPOSE: A new transtentorial venous system consisting of medial, intermediate, and lateral tentorial veins, connecting infra- and supratentorial compartments, was recently shown in 2 cadaver dissections and 2 patient scans. We sought to characterize the venous patterns within the tentorium and their relation to measures of skull development in a cohort of healthy adults.

MATERIALS AND METHODS: We retrospectively reviewed tentorial venous anatomy of the head using CTA/CTV performed for routine care or research purposes in 238 patients. Included studies had adequate contrast opacification of venous structures and a section thickness of $\leq 2 \mathrm{~mm}$; we excluded cases with space-occupying lesions and vascular pathologies. Tentorial angle, dural sinus configurations, and measures of skull base development were assessed as predictors of tentorial venous anatomy variation via Cramér $\mathrm{V}$ association, the binary encoded Pearson correlation, and nearest-point algorithm with the Euclidean distance metric for clustering.

RESULTS: Tentorial vein development was related to the ringed configuration of the tentorial sinuses $(P<.005)$. There were 3 configurations. Groups 1A and 1B $(n=50 / 238)$ had ringed configuration, while group 2 did not ( $n=188 / 238)$. Group 1A ( $n=38 / 50)$ had a medialized ringed configuration, and group $1 \mathrm{~B}$ had a lateralized ringed configuration $(n=12 / 50)$. Measurements of skull base development were predictive of these groups. The ringed configuration of group 1 was related to the presence of a split confluens, which correlated with a decreased internal auditory canal-petroclival fissure angle. Configuration $1 \mathrm{~A}$ was related to the degree of petrous apex pneumatization ( $P$ value $=.010)$.
\end{abstract}

CONCLUSIONS: Variations in the transtentorial venous system directly correlate with cranial development.

ABBREVIATIONS: CC-JB = carotid canal-jugular bulb; IAC-PCF = internal auditory canal-petroclival fissure; ITV = intermediate tentorial vein; LTS = lateral tentorial sinus; LTV = lateral tentorial vein; MTS = medial tentorial sinus; MTV = medial tentorial vein; RC = ringed configuration

$\mathbf{N}$ euroradiologists are critical in presurgical planning, particularly for complex surgical approaches to the skull base such as anterior petrosectomy, combined presigmoid/retrosigmoid approaches, and supracerebellar-transtentorial approach to the mesial temporal lobe. ${ }^{1-5}$ The safety of these surgical approaches has been framed around incising avascular regions of the tentorium. However, tentorial sectioning may still produce unexplained adverse consequences. ${ }^{6,7}$ Several cadaveric and radiographic studies

Received April 2, 2020; accepted after revision July 2.

From the Surgical Neurology Branch (J.S.R., J.D.H.), National Institute of Neurological Disorders and Stroke, National Institutes of Health, Bethesda, Maryland; Neuro-Oncology Branch (J.S.R.), National Cancer Institute, National Institutes of Health, Bethesda, Maryland; Department of Radiology and Radiological Science (J.M.T., C.W.), The Johns Hopkins University School of Medicine, Baltimore, Maryland; SmartSulis LLC (V.C.), Trabuco Canyon, California; Section on Medical Neuroendocrinology (A.J.), Eunice Kennedy Shriver National Institute of Child Health and Human Development, National Institutes of Health, Bethesda, Maryland; Department of Neurosurgery (M.N.), Hospital Beneficência Portuguesa de São Paulo, Institute of Neurological Science of São Paulo Bela Vista, São Paulo, Brazil; Department of Radiology (I.S.), George Washington University, Washington, DC; MedPix (J.S.), National Library of Medicine, Bethesda, Maryland; and Department of Neurosurgery (B.R.R.), Riverview Medical Center, Red Bank, New Jersey.

previously demonstrated the anatomic patterns of bridging veins to the tentorium and medial and lateral venous sinuses within the tentorium. ${ }^{8-11}$ A recent study found a relationship between the anatomy of the tentorial sinuses at the transverse-sigmoid junction and cranial morphometrics. ${ }^{12}$ However, several studies of veins within the tentorium have demonstrated a wide variation inconsistent

Paper previously presented as an E-poster and abstract at: Annual Meeting of the American Association of Neurological Surgeons, April 25-29, 2020; Boston, Massachusetts.

This study was supported, in part, by the Intramural Research Programs of the National Institute of Neurological Disorders and Stroke and National Cancer Institute at the National Institutes of Health.

Please address correspondence to Jared S. Rosenblum, MD, Building 35, Room 2B203, Neuro-Oncology Branch, Center for Cancer Research, National Cancer Institute, Bethesda, MD 20892; e-mail: Jared.rosenblum@nih.gov; (j)aredRosenblum

\footnotetext{
- Indicates open access to non-subscribers at www.ajnr.org Indicates article with supplemental on-line appendix and tables. Indicates article with supplemental on-line photos. http://dx.doi.org/10.3174/ajnr.A6775
} 
with direct drainage of the bridging veins into the tentorial sinuses. ${ }^{13-16}$ Intimate knowledge of the normal variations of the tentorial venous anatomy is of critical importance for neuroradiologists guiding neurosurgeons in presurgical planning and understanding postoperative complications.

Recently, a transtentorial venous system comprising the tentorial sinuses and veins connecting the anterior, middle, and posterior fossae was identified in 2 cadavers without intracranial pathology and 2 patients with disparate outcomes: 1) remote cerebellar infarct and hemorrhagic conversion due to compromise of the transtentorial venous system, and 2) tolerance of bilateral transverse sinus thrombosis because of collateral drainage through the tentorial veins. ${ }^{17}$ Thus, the tentorial veins, remnants of the embryologic venous drainage system, ${ }^{18-20}$ appeared to retain pathologic and physiologic relevance. ${ }^{17}$

The transtentorial network comprises 3 main veins and 2 main tentorial sinuses. The medial and lateral tentorial sinuses (MTS and LTS) may be distinct or connected, described previously as the ringed configuration (RC). ${ }^{14,15}$ The medial tentorial vein (MTV) courses within the free edge of the tentorium, originating from the straight sinus and draining to the cavernous sinus. The intermediate tentorial vein (ITV) courses within the tentorium from the straight sinus to the superior petrosal sinus. The lateral tentorial vein (LTV) originates from the MTS or the RC of the tentorial sinuses and drains to the superior petrosal sinus. Plexiform anastomoses connect the tentorial veins and sinuses at various points. ${ }^{17}$

Herein, we aimed to define normal variations in the tentorial venous anatomy within the general population. Further, we hypothesized that the variations in tentorial sinus and vein anatomy were related to and could be predicted by the extent of skull base development, as measured by cranial morphometrics.

\section{MATERIALS AND METHODS Study Criteria}

Tentorial venous anatomy of the head was evaluated on CTA and CTV performed for routine care or for research during 1 year at a single institution (The Johns Hopkins Hospital). Patients underwent vascular imaging either for research protocol, stroke evaluation, or follow-up of known lesions.

Studies were reviewed by expert neuroradiologists and the study team. Patients with known space-occupying lesions or vascular pathologies including acute dural venous sinus stenosis or thrombosis, AVM/AVF, new aneurysms or those enlarging on follow-up, cavernous malformations, developmental venous anomalies, intracranial hemorrhage, or infarction were excluded. Patients with stable aneurysms $(n=9 ; 3>4 \mathrm{~mm})$ with a long duration of follow-up were included; no change in intracranial vascular anatomy was seen longitudinally in these patients. We included studies with adequate contrast opacification of the venous structures and section thicknesses of $\leq 2 \mathrm{~mm}$.

The study protocol for retrospective chart review was approved by the institutional review board.

\section{Radiologic Measures}

Measurements of the Skull Base. Scans were performed on 64-section multidetector CT (Definition 64 AS Section; Siemens) with $120 \mathrm{kV}, 175 \mathrm{~mA}, 64 \times 0.6 \mathrm{~mm}$ collimation, after IV nonionic contrast (Omnipaque 350 [iohexol]or Visipaque 320 [iodixanol]; GE Healthcare). Axial $0.75-\mathrm{mm}$ slices at increments of $0.5 \mathrm{~mm}$ were reconstructed using a $512 \times 512$ matrix, with a standard kernel.

Bony measurements were performed on CT to assess skull base development, as follows. The petrosagittal angle was measured at the intersection of a midsagittal line from the nasal septum to the internal occipital protuberance and a line on each side from the spheno-occipital synchondrosis to the stylomastoid foramen. ${ }^{21}$ The angle between the internal auditory canal and the petroclival fissure (IAC-PCF angle) was also measured. ${ }^{22}$ The distance between the carotid canal and jugular bulb (CC-JB distance) was measured $1 \mathrm{sec}-$ tion before the turn of the petrous segment of the internal carotid artery. ${ }^{22}$ Development of the petrous apex was assessed as petrous apex pneumatization. ${ }^{22}$ The tentorial angle was measured between a line extending from the nasion through the tuberculum sella and a second line through the straight sinus in the midsagittal plane. ${ }^{23}$ Sample measurements are shown in On-line Fig 1. Measurements were performed by the study team on OsiriX DICOM Viewer (https://www.osirix-viewer.com/).

Definition of Dural Sinuses, Tentorial Sinus, and Tentorial Vein Anatomy. The transverse sinuses were assessed for patency and congenital stenosis. The cavernous sinuses were also assessed for patency or developmental anomalies. Congenital anomaly was distinguished from new stenosis by reviewing longitudinal imaging when available or by correlating with clinical findings-such as papilledema or elevated intracranial pressure; these cases were excluded. The presence of a split confluens sinuum was also noted. The tentorial sinuses were defined medial or lateral along the tentorium and by their presence and respective origins. ${ }^{8}$ The MTS either originates from the straight sinus or the medial portion of the transverse sinus. ${ }^{8}$ The LTS, when present, originates from either the lateral portion of the transverse sinus or the transverse-sigmoid sinus junction. ${ }^{8,11}$ The RC of the tentorial sinuses was defined as a continuity of the MTS and LTS. ${ }^{13,15}$ The MTV, ITV, and LTV were defined as previously described. ${ }^{17}$

\section{Volumetric Reconstruction of CT Venography}

Volume-rendered 3D images were generated using SkyScan CT-Vox software. DICOM study files were imported into CTVox in DICOM 8-bit format (https://www.bruker.com/products/ microtomography/micro-ct-software/3dsuite.html). The preview function in CTVox was used to select an appropriate section with the contrast-enhanced structures of interest, eg, tentorial veins. For the patient shown in Figure 1, this was coronal section IM-021-074; DICOM files are available in the Supplementary DICOM Data (https:/gin.g-node.org/JaredRosenblum/ Rosenblum_AJNR_2020_Supplemental_DICOM_Data_and_ Transfer_Function). The initial suggested dynamic range for this study in the 8-bit data converter was 1034-1134 $\mathrm{HU}$ with a minimum of 0 and a maximum of $65,535 \mathrm{HU}$. The highest intensity pixel value of the chosen section for this scan was $2580 \mathrm{HU}$.

The import histogram was restricted to exclude parenchyma, saturate contrast within the tentorial veins, and to lessen saturation of bone. The lower bound was set to $1075 \mathrm{HU}$ to exclude parenchyma, which was approximately $40 \mathrm{HU}$. The upper bound was set to $3580 \mathrm{HU}$, which was $1000 \mathrm{HU}$ above the maximum value 
of bone. The opacity was adjusted to show a limited view of the surrounding soft tissues and highlight vessels with contrast. This was saved as a preset transfer function that can be loaded into CTVox (Supplementary Transfer Function). Lighting was adjusted to optimize visualization of the render; $18 \%$ shadows, $0 \%$ specular, and $100 \%$ diffuse settings were applied.

\section{Statistical Analysis}

Determining Variable Relationships. Categories of dural sinuses and tentorial veins were defined on the basis of the definitions of the anatomy above. Cramér V association estimator (https://github. com/shakedzy/dython/blob/master/dython/nominal.py), which depends on $\chi^{2}$ comparisons, was used to measure the association strength among categoric variables based on the above definitions, holding values between 0 and $1 .^{24}$ This determines whether there is a significant difference between expected and observed frequencies in the categories. The equation for strength of association is

$$
V=\sqrt{\frac{x^{2} / n}{\min (c-1, r-1)}},
$$

where $n=$ sum total of observations, $c=$ number of columns, and $r=$ number of rows.

Preliminary categories were made on the basis of observations in the patient population. To incorporate all variables into the Cramér $\mathrm{V}$ estimation and validate categories, we binned numeric variables into ranges. These included all bony angular and distance measurements and the tentorial angle.

Related variables (categories) were clustered and then linked using the nearest-point algorithm, ${ }^{25,26}$ which computes the minimum (min), two elements, one in each cluster, that are closest to each other, distances (dist) using the following equation:

$$
d(u, v)=\min [\operatorname{dist}(u[i], v[j])]
$$

for all points $i$ in cluster $u$ and $j$ in cluster $v$. The distance metric used was the Euclidean distance, ${ }^{27,28}$ which arranges all points as $m n$-dimensional vectors. Agglomerated variables were highlighted through a dendrogram heat map, which represents both the strength of relationships and variable relationship clusters. To validate relationship clusters, we performed uncertainty coefficient analysis (Thiel's $U$ association measure), which is derived from the conditional entropy between 2 variables. ${ }^{29}$

To perform correlation analysis and determine the directionality of relationships across all variables, we binarily encoded categoric variables into dummy variables. Each categoric value was assigned a new column, assigning a 1 or 0 to each sample, depending on the presence or absence of that specific value.

To measure the direction of linear relationship between numeric variables, we used the Pearson correlation. ${ }^{28}$ The equation is

$$
\rho=\frac{\operatorname{cov}(X, Y)}{\sigma_{x} \sigma_{y}}
$$

and is calculated in a sample population as

$$
r=\frac{\sum_{i=1}^{n}\left(x_{i}-\bar{x}\right)\left(y_{i}-\bar{y}\right)}{\sqrt{\sum_{i=1}^{n}\left(x_{i}-\bar{x}\right)^{2}\left(y_{i}-\bar{y}\right)^{2}}} .
$$

The Pearson correlation coefficient holds values between -1 and 1 . Negative values imply an inverse linear relationship; positive values imply a positive linear correlation. Variables were clustered and linked using the nearest-point algorithm, with Euclidean distance as the distance metric. Correlated and clustered variable outcomes were highlighted through a dendrogram heat map. Observation distributions were plotted for all variables. The kernel density estimate and histograms were used to visualize probability distributions.

\section{RESULTS}

Of the available radiologic imaging of the head at our institution, $238 \mathrm{CTA} / \mathrm{CTV}$ studies were included in the investigation based on the criteria defined in the study criteria section of the Materials and Methods. Patient data are available in the On-line Supplementary Data. Patients ranged in age from 19 to 100 years, with a mean age of $58 \pm 18$ years; this is summarized in the On-line Summary Statistics file. Delayedphase CTA or CTV was optimal for evaluating the tentorial veins and was also used for $3 \mathrm{D}$ reconstruction and visualization (Fig 1). The tentorial sinuses were observed in their known variations, including the RC. We confirmed the common presence of the MTV, ITV, and LTV and found variations (Fig 2). The MTV was always present in the expected location along the tentorial free edge. The ITV and LTV had variable configurations.

Preliminary categories of these variations were defined (Online Table 1) for statistical analysis. Detailed patient characteristics and category distribution are provided in On-line Fig 2. The ITV and LTV were categorized according to their interruption and drainage patterns. The baseline category (variation 0 ) was a continuous and uninterrupted LTV, as previously described. ${ }^{17}$ The ITV was further categorized into 4 variations. Variation 1 was interrupted by the MTS, draining from the straight sinus to the superior petrosal sinus. Variation 2 was interrupted by the LTS, draining from the straight sinus to the superior petrosal sinus. Variation 3 was interrupted by a plexiform anastomosis, draining from the straight sinus to the MTV. Variation 4 was interrupted by the RC, draining to the superior petrosal sinus. The LTV was similarly categorized as follows. In variation 1 , the LTV was interrupted by the LTS, draining from the transverse sinus to the superior petrosal sinus. In variation 2 , the LTV was interrupted by a lateralized $\mathrm{RC}$, draining from the transverse sinus to the RC. In variation 3, the LTV was interrupted by a medialized RC, draining from the RC to the superior petrosal sinus.

The Cramér V association clustered heat map showed 2 intercorrelated variable groupings (Fig 3). The first group consisted of the following variables: the origin of the MTS, the presence or absence of the RC of the tentorial sinuses, and the configurations of the ITV and LTV. The second group consisted of the cavernous sinus patency or congenital anomaly, transverse sinus patency or congenital stenosis, origin of the LTS, and the presence of the MTV. The uncertainty coefficient (Thiel's U) confirmed the clustering groups and provided directionality to the associations (On-line Fig 3). 

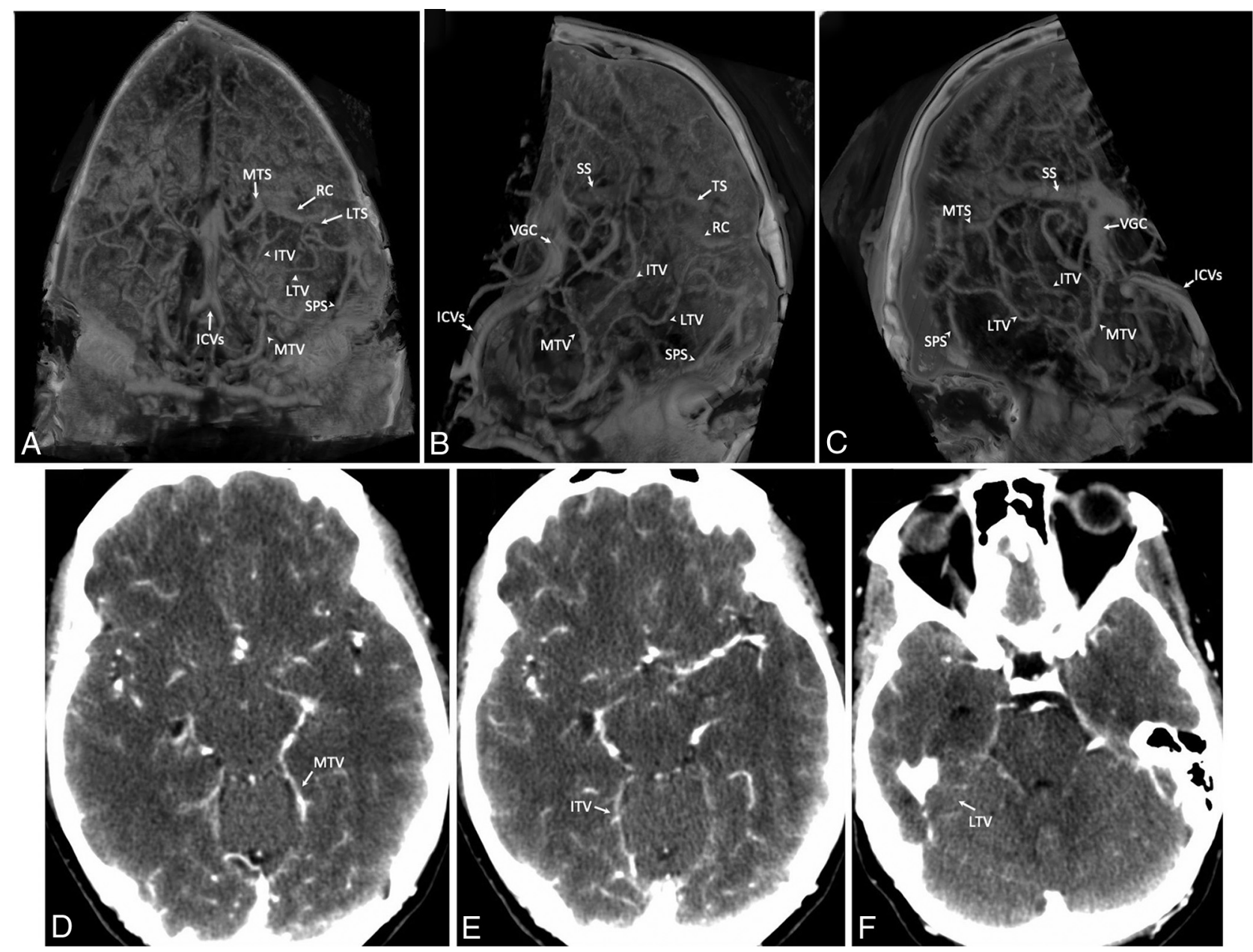

FIG 1. Transtentorial veins. A-C, 3D volumetric reconstruction of delayed-phase CTA of the head and neck; parenchyma (0-40 HU) has been segmented out, leaving the intracranial vessels filled with contrast. $A$, Reconstructed volume of the head is shown from the anteroposterior view with a $30^{\circ}$ downward rotation to show the plane of the tentorium. $B$, The same reconstruction as in $A$ is rotated to show the left side. $C$, The same reconstruction rotated to show the right side, which is symmetric. $D-F$, Source axial images for the volumetric reconstruction of this scan are shown; MTV, ITV, and LTV are labeled. ICV indicates the internal cerebral vein; SS, straight sinus; SPS, superior petrosal sinus; VGC, vein of Galen confluens; TS, transverse sinus; MTV, medial tentorial sinus.

A

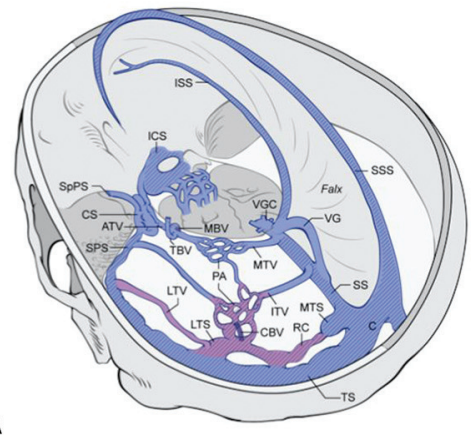

B

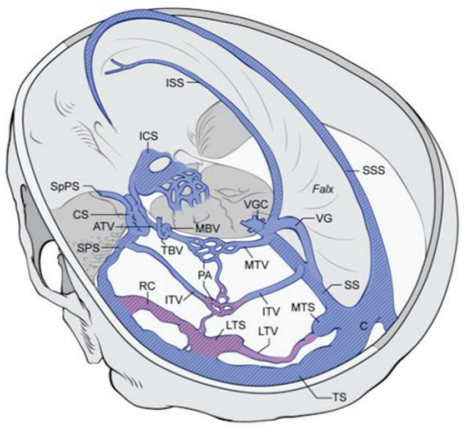

C

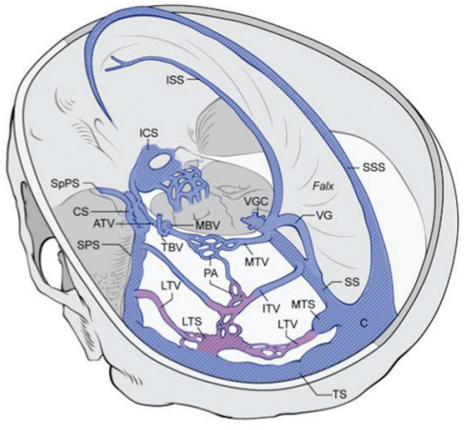

FIG 2. Schematic representation of 3 transtentorial vein configurations. The 2 groups of tentorial sinus configurations are shown; group 1 , which is subdivided, has an RC, while group 2 does not. Regions of variation are shaded in purple, cross-hatching denotes sinuses, and veins are blue. The split confluens is shown for groups $1 \mathrm{~A}$ and $\mathrm{BB}$. The degree of petrous apex pneumatization for each group is also shown. A, Configuration $1 \mathrm{~A}$ has a medialized RC, and the ITV is interrupted by plexiform anastomosis to the RC or LTS. B, Configuration IB has a lateralized RC, and the ITV is uninterrupted. C, In configuration 2, the RC is absent; the LTV connects the MTS to the LTS and the LTS to the superior petrosal sinus. Relevant draining sinuses are also shown. CS indicates cavernous sinus; ATV, apical tentorial vein; CBV, cerebellar bridging vein; ICS, intercavernous sinus; ISS, inferior sagittal sinus; MBV, mesencephalic bridging vein; PA, plexiform anastomosis; SpPS, sphenoparietal sinus; SS, straight sinus; SSS, superior sagittal sinus; VGC, vein of Galen confluens; VG, vein of Galen; TS, transverse sinus; SPS, superior petrosal sinus; C, confluens; TBV, tentorial bridging vein; ICV, internal cerebral vein. 
Cramér V Association

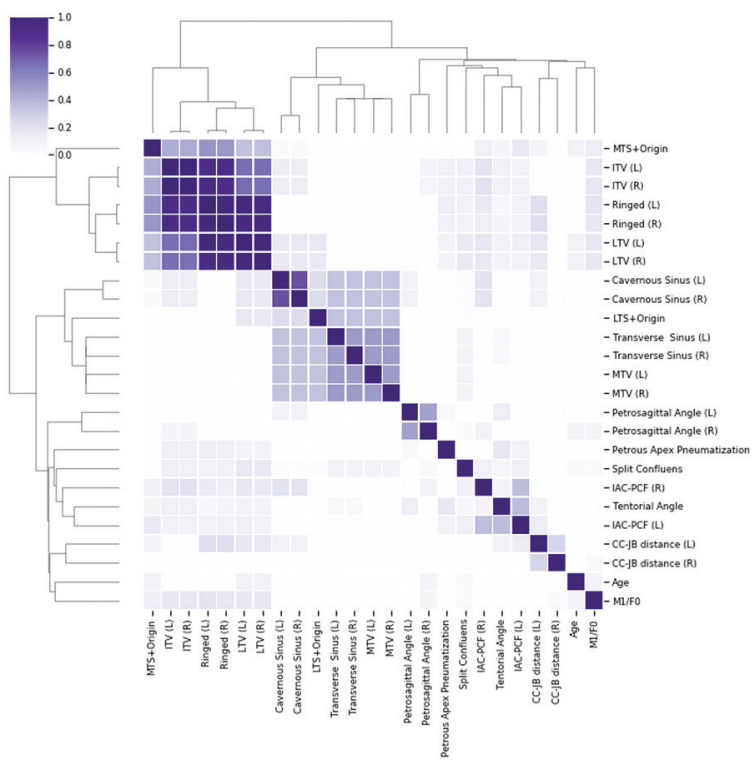

Pearson Correlation

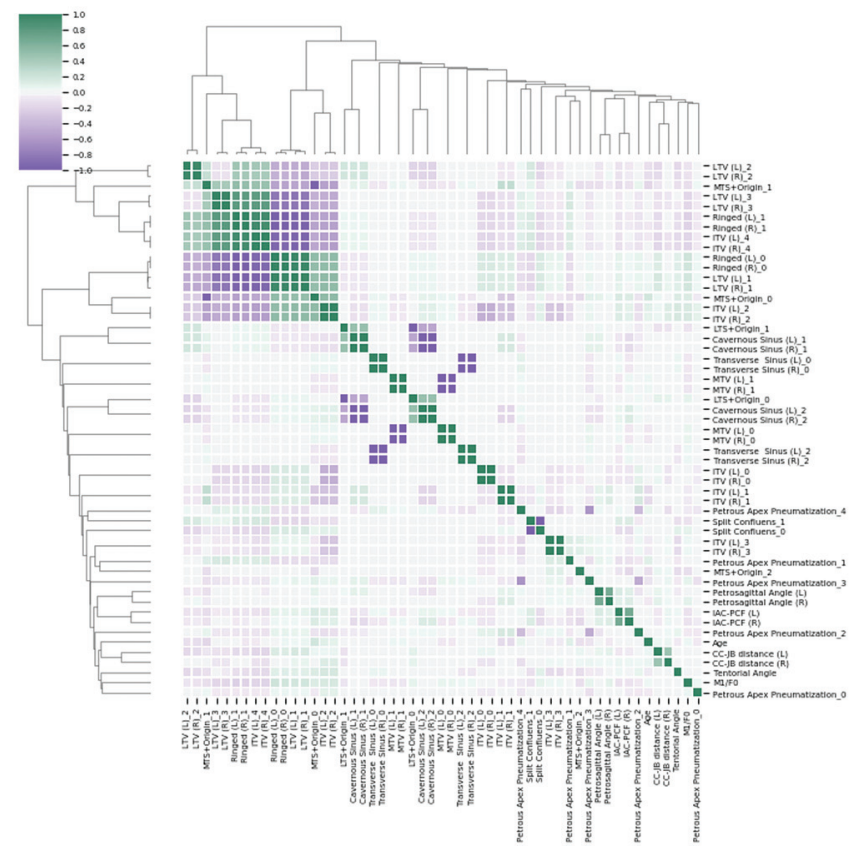

FIG 3. Association and correlation with clustering highlights common tentorial vein configurations. Left, Cramér $V$ association heat map, a symmetric measure of associations among categoric variables, presents 2 intercorrelated variable groupings. The first shows a high-to-perfect association among the MTS origin, RC, ITV, and LTV. The second shows a moderate-to-high association among the cavernous sinus, LTS origin, transverse sinus, and MTV. Right, The Pearson correlation heat map reveals 2 primary configurations, one of which also has an alternate configuration based on the presence or absence of the ring connection of the MTS and LTS. In the first configuration, the RC is medialized. Due to the presence of the medialized RC originating from the MTS at the straight sinus, the tentorial veins have the following connections: The ITV is interrupted by the RC, and the LTV is lateralized, draining to the superior petrosal sinus. An alternate and slightly less common configuration comprises a lateralized RC and resultant medialized LTV. The second primary configuration has no RC, the ITV is interrupted by the LTS, and the LTV is also interrupted by the LTS. In this configuration, the MTS may be present or absent. IAC-PCF (left) and (right) (degrees); petrosagittal angle (left) and (right) (degrees); CC-JB distance (left) and (right) (millimeters); remaining variables are categoric. The ITV was categorized as 0 through 4 as per the result of preliminary categorization. LTV was categorized as 1 through 3 per results of preliminary categorization. female $=0 ; \mathrm{Ml}$, male $=1$.

The Pearson correlation clustered heat map for all variables (Fig 3) performed to further assess the directionality of relationship between the correlated variables showed 2 primary groups based on the presence or absence of the RC of the tentorial sinuses ( $P$ values shown in On-line Tables 2 and 3). The first group was further divided into subgroups based on the location of the RC. Groups $1 \mathrm{~A}$ and $1 \mathrm{~B}(n=50 / 238)$ had RC, while group 2 was defined by its absence $(n=188 / 238)$. Configuration $1 \mathrm{~A}(n=38 / 50)$ had a medialized RC tentorial sinus, while it was lateralized in configuration 1B $(n=12 / 50)$. Further, measures of skull base development were predictive of these configurations (Fig 4). The RC of group 1 was related to the presence of a split confluens, which was predicted by a decreased IAC-PCF angle (Fig 5). Configuration 1A was related to the degree of petrous apex pneumatization.

\section{DISCUSSION}

This study evaluated the anatomy of the tentorial sinuses and veins in a large healthy population using CTA/CTV $(n=238)$ and thus identified patterns of variation of the tentorial veins. Further, we evaluated the relationships among cranial morphometrics, dural and tentorial sinuses anatomy, and tentorial venous anatomy. Using statistical analyses, we identified bony measurements that can predict the configurations of tentorial veins, including the IAC-PCF angle and the degree of petrous apex pneumatization that can be made on CT/CTA/CTV. In addition, we developed a semiautomated process of volumetric reconstruction of dural and tentorial venous anatomy used in this study. Together, our study provides information for radiographic evaluation of intracranial venous anatomy critical for surgical planning and avoidance of complications that may arise as a consequence of sacrifice of tentorial veins. ${ }^{6,17}$ Further, our study may aid in determining which patients with postoperative venous sinus thrombosis remain asymptomatic or resolve spontaneously versus which require intervention. ${ }^{7}$ Knowledge of the transtentorial venous system is critical to neuroradiologists guiding neurosurgeons in surgical planning of skull base approaches. Further, this anatomy will enable prompt neuroradiologic recognition of surgical complications.

Although we did not perform a head-to-head comparison, the MR imaging, MRA, and MRV studies available at our institution were excluded from analysis because of poor visualization of the structures of interest. This was either due to larger section thickness, lack of contrast opacification of small-caliber vessels, or noncontrast (time-of-flight) measurement, which did not adequately demonstrate tentorial veins. Thus, this study included only CTA/CTV with a section thickness of $0.75 \mathrm{~mm}$. From these studies, we identified 2 primary groups of tentorial venous anatomy in this population. These groups were correlated to the RC of the tentorial sinuses. Group $1 \mathrm{~A}$, which was characterized by a 

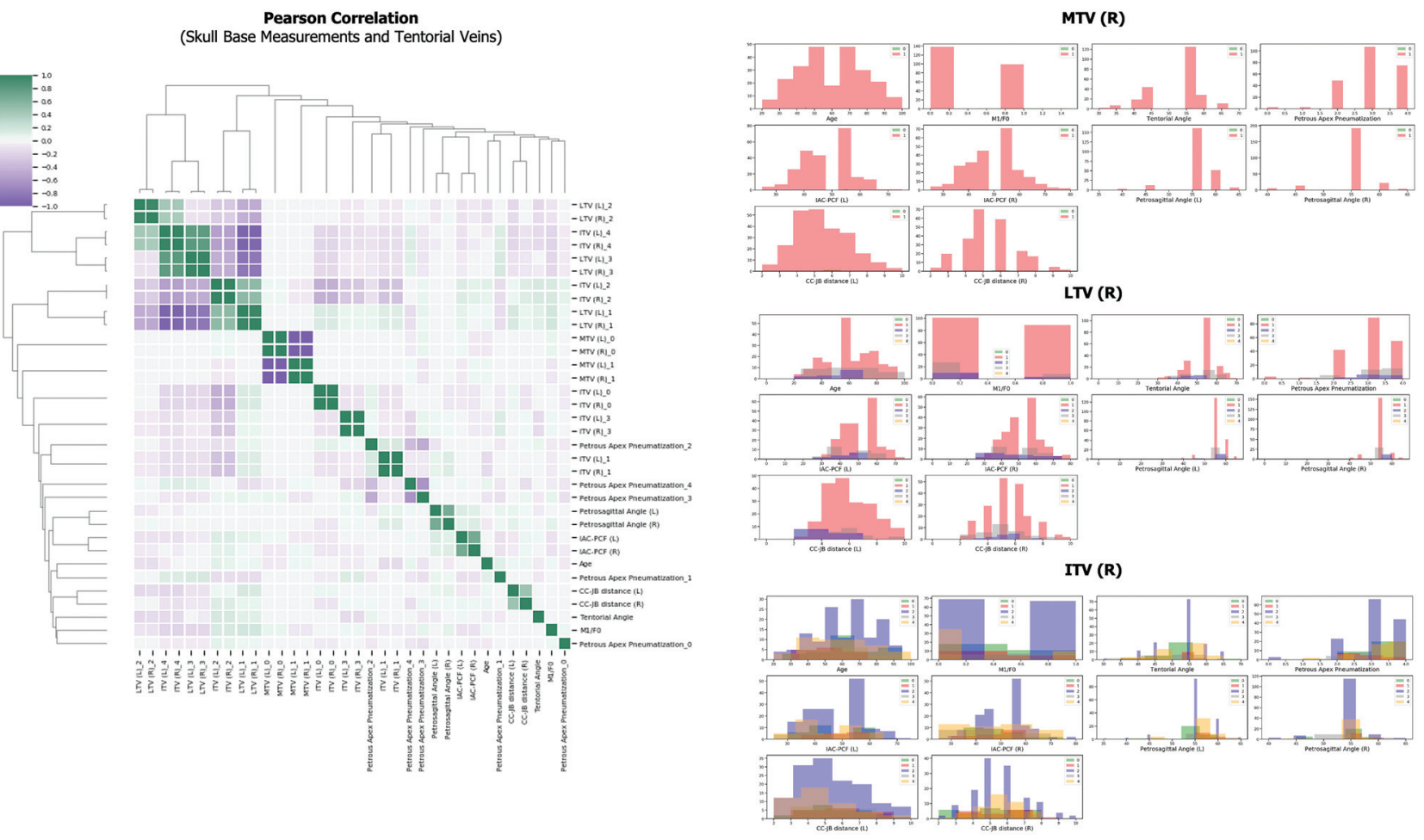

FIG 4. Tentorial vein and skull base measurement subset correlation with clustering and distribution plots. Left, The Pearson correlation heat map further characterizes the medialized RC with the ITV and LTV. This characterization confirms that when the RC of the MTS and LTS is present and medialized, the ITV and LTV originate from this connection and are lateralized (group 1A). The strongest associated configuration comprises this; the lateralized RC is confirmed (group 1B). The configuration with the absent RC between the tentorial sinuses is also again confirmed (group 2). This configuration appears related to the CC-JB distance and the tentorial angle in the heat map. Right, Y-axes in all charts are patient numbers. Probability density functions and kernel density estimates for skull base measurements, grouped by tentorial vein categories, reveal additional relationships. The distribution plots are shown for the right side of the tentorium only because symmetry is upheld as shown in the heat map. The probability density functions for LTV 3 and ITV 4 (configuration IA) are nearly identical; this is inversely related to male sex, CC-JB distance, and petrous apex pneumatization 0 (less complete). This group is positively correlated to petrous apex pneumatization 1 (more complete). Group 1B has no correlation with petrous apex pneumatization; however, it is distinguished from group 2 by oppositional correlation to the IAC-PCF. The probability density functions and kernel density estimates of ITV 2 and LTV 1 (group 2) are very similar. IAC-PCF (left) and (right) (degrees); petrosagittal angle (left) and (right) (degrees); CC-JB distance (left) and (right) (millimeters); the remaining variables are categoric. ITV was categorized as 0 through 4 per the results of preliminary categorization. LTV was categorized as 1 through 3 per the results of preliminary categorization. $\mathrm{FO}$ indicates female $=0 ; \mathrm{Ml}$, male $=1 ; \mathrm{R}$, right; $\mathrm{L}$, left.

medialized RC, and group $1 \mathrm{~B}$, which was characterized by a lateralized RC, were correlated to the presence of a split confluens, a proposed sign of incomplete late development of the dural sinuses. ${ }^{30}$ Conversely, group 2 (characterized by the absence of the RC) was correlated with complete development of the confluens. Further, these groups were predicted by bony measures of skull base development, such as the degree of petrous apex pneumatization or the IAC-PCF angle. Thus, the cranial morphometrics performed predicted specific intracranial venous configurations.

These findings are consistent with previous investigations of venous development. First, tentorial drainage of the midbrain veins is critical during development. ${ }^{17,30}$ Second, at this time, when the jugular foramen is narrowed by the developing petrous pyramid, retrograde venous flow dilates the transverse sinuses. ${ }^{30}$ The dilation of the transverse and sigmoid sinuses results in the their pouching into the tentorium and subsequent development of the medial and lateral tentorial sinuses and cortical veins, including the vein of Labbé, which may drain into these. ${ }^{30}$ Extracranial drainage of these sinuses, including the mastoid, anterior condylar, and posterior condylar emissary veins, also develops during the dilation phase and most often originates from the sigmoid sinus. ${ }^{30}$ Third, joining of the 2 transverse sinuses into the confluens is the last part of development of the dural sinuses. ${ }^{18,30}$ The transverse sinuses, petrosal sinuses, and cavernous sinuses develop from the lateral head vein on each side. ${ }^{31}$ We hypothesized on the basis of this information that the tentorial venous drainage pattern would depend on the extent of development of the skull base.

Grouping our observations in this patient population by categories such as bony measures, dural sinuses, and tentorial veins generated preliminary categoric relationships via the Cramér V association measure. For example, without tentorial sinuses in the $\mathrm{RC}$, both the ITV and LTV can originate from the straight sinus. However, in the presence of a medialized RC, such as in group 1A, the LTV, and less often the ITV, may originate from the RC. These relationships were grouped into putative anatomic configurations of venous drainage via an agglomerative clustering algorithm and dendrogram heat maps. These intercorrelated variable groupings were confirmed by the Thiel's $U$ association. Because the uncertainty coefficient is not a symmetric analysis, it allows understanding of the conditionality behind relationships. For example, 

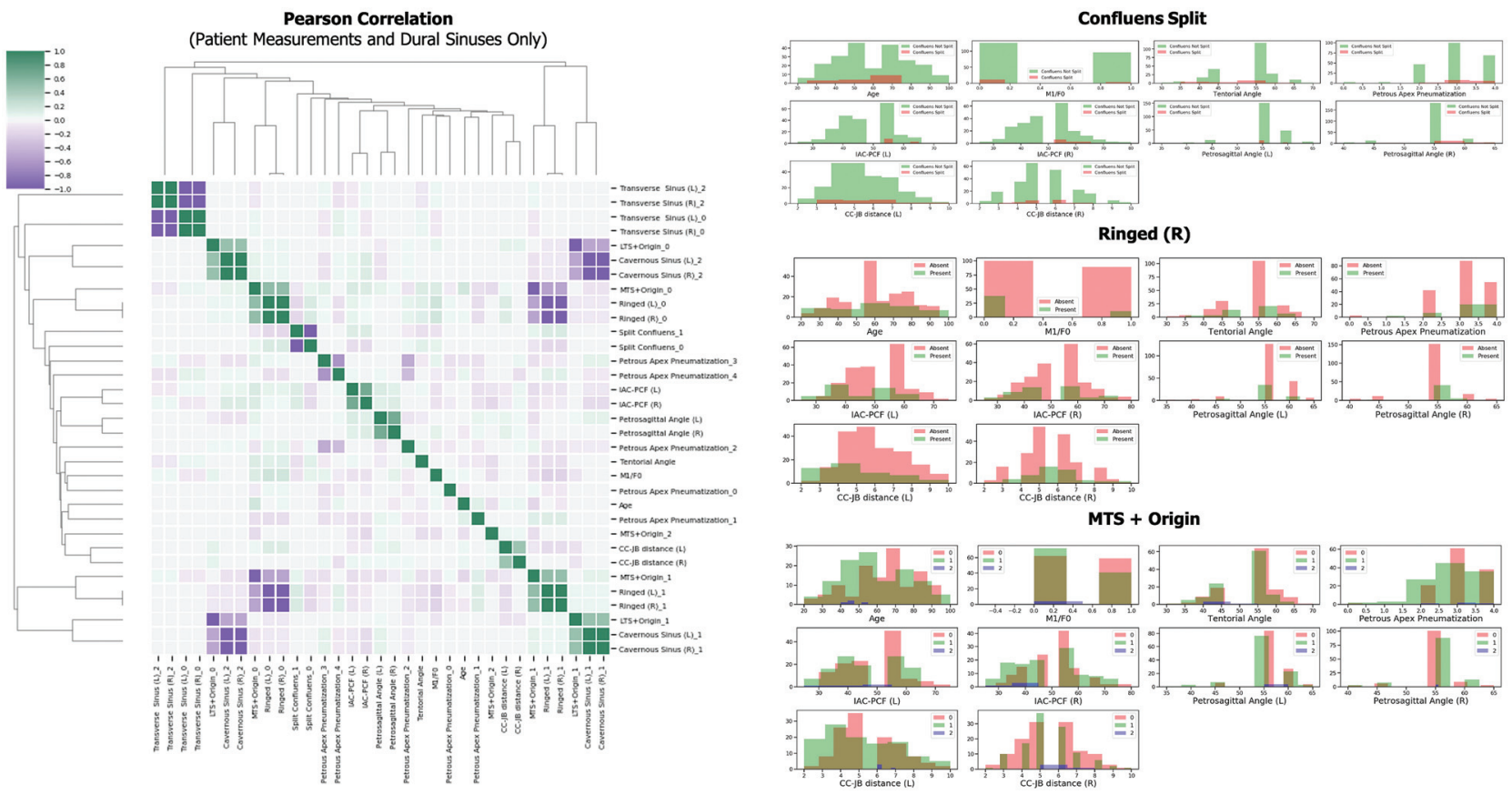

FIG 5. Dural sinus and skull base measurement subset correlation with clustering and distribution plots. Left, The Pearson correlation heat map further characterizes the relationship among the dural sinuses, cavernous sinuses, and skull base measurements. This confirms that an LTS originating in the transverse sinus is linked with a patent cavernous sinus. As a corollary, an LTS originating in the transverse sinus-sigmoid sinus junction is linked with a diminutive cavernous sinus. The clustering also reiterates the relationship between the RC presence and the location of the origin of the MTS. Right, Y-axes in all charts are patient numbers. Probability density functions and kernel density estimates for skull base measurements, grouped by dural sinus categories, provide additional support for the determined anatomic configurations. The distribution plots are shown for the right side of the RC only because symmetry was upheld as shown in the heat map. The probability density functions for LTS originating from the transverse sinus (LTS origin 0 ) and patent cavernous sinus (cavernous sinus 2) are similar; these show a weak positive correlation with the absent RC (configuration 2). Conversely, the probability density functions for LTS originating at the transverse sinus-sigmoid sinus junction (LTS origin 1) and a congenitally diminutive cavernous sinus (cavernous sinus 1) are similar, showing a weak positive correlation with the RC (configuration family 1). IAC-PCF (left) and (right) (degrees); petrosagittal angle (left) and (right) (degrees); CC-JB distance (left) and (right) (millimeters); the remaining variables are categoric. The ITV was categorized as 0 through 4 per the results of preliminary categorization. The LTV was categorized as 1 through 3 per the results of preliminary categorization. F0 indicates female $=0 ; M 1$, male $=1$; R, right; L, left.

knowing the RC status allows strong prediction of the ITV and LTV configurations; however, conversely, knowing the ITV category is a weaker predictor of RC status.

Subsequent binary encoding of variables, the Pearson correlation, and clustering confirmed and further defined the putative anatomic configurations. For example, the most common cluster in the putative groups comprised the following variables: the presence or absence of RC, origin of the MTS, ITV configuration, and LTV configuration. These held true for the Pearson correlation, which provided further detail and clarified the 3 groups defined above: groups $1 \mathrm{~A}, 1 \mathrm{~B}$, and 2 .

The statistical analyses performed in this study not only allowed the confirmation of the presence of the transtentorial venous system but also identified the relationship between completed development of the skull base and the configurations of this venous drainage. Knowledge of the venous anatomy of the tentorium is critical for surgical planning of approaches involving manipulation or sectioning of the tentorium or veins bridging to it. Further, this transtentorial venous system may explain certain phenomena, including remote cerebellar infarction and asymptomatic spontaneous or postoperative transverse sinus thrombosis.

While this study did not evaluate patients undergoing surgical intervention, previous studies have described or hypothesized venous configurations that may lead to adverse outcomes such as remote cerebellar infarct. ${ }^{11,17}$ Our analysis supports the importance of avoiding these complications by preserving both the MTV and LTS and their sole venous drainage of remote parenchyma. We hypothesize that configurations with greater anastomoses between tentorial veins, such as Group 1A found in this study, would be less prone to complications of sectioning the tentorium. However, the tentorial venous drainage must be considered in the context of the intracranial venous drainage, such as a narrowed transverse sinus or jugular bulb, as previously shown to lead to complication. ${ }^{17}$ Further study of the clinical and surgical relevance of this transtentorial venous system is needed.

\section{CONCLUSIONS}

The analysis of 238 patients identified 3 variations of the transtentorial venous system in a large healthy population and identified bony measures of skull base development associated with each variation. Knowledge of this anatomy is critical for neuroradiologists and neurosurgeons in surgical planning, reducing the risk of interventions, and explaining the mechanism of surgical complications arising from compromise of the tentorial venous system.

AJNR Am J Neuroradiol 41:1825-32 Oct 2020 www.ajnr.org 


\section{ACKNOWLEDGMENT}

The authors would like to acknowledge the National Institutes of Health Medical Arts Department, specifically Erina He, for the schematic representation in Fig 2.

\section{REFERENCES}

1. Kawase T, Shiobara R, Toya S. Middle fossa transpetrosal-transtentorial approaches for petroclival meningiomas. Selective pyramid resection and radicality. Acta Neurochir (Wien) 1994;129:113-20 CrossRef Medline

2. Al-Mefty O, Ayoubi S, Smith RR. The petrosal approach: indications, technique, and results. Acta Neurochir Suppl (Wien) 1991:53:166-70 CrossRef Medline

3. Malis LI. Surgical resection of tumors of the skull base. In: Wilkins RH, Rengachary SS, eds. Neurosurgery. Vol 1. McGraw-Hill; 1985:1011-21

4. Türe U, Harput MV, Kaya AH, et al. The paramedian supracerebellar-transtentorial approach to the entire length of the mediobasal temporal region: an anatomic and clinical study: laboratory investigation. J Neurosurg 2012;116:773-79 CrossRef Medline

5. de Oliveira JG, Párraga RG, Chaddad-Neto F, et al. Supracerebellar transtentorial approach-resection of the tentorium instead of an opening-to provide broad exposure of the mediobasal temporal lobe: anatomical aspects and surgical implications. J Neurosurg 2012;116:764-72 CrossRef Medline

6. Amini A, Osborn AG, McCall TD, et al. Remote cerebellar hemorrhage. AJNR Am J Neuroradiol 2006;27:387-90 Medline

7. Benjamin CG, Sen RD, Golfinos JG, et al. Postoperative cerebral venous sinus thrombosis in the setting of surgery adjacent to the major dural venous sinuses. J Neurosurg 2018 Oct 1. [Epub ahead of print] CrossRef Medline

8. Matsushima T, Suzuki SO, Fukui M, et al. Microsurgical anatomy of the tentorial sinuses. J Neurosurg 1989;71:923-28 CrossRef Medline

9. Oka K, Rhoton AL Jr, Barry M, et al. Microsurgical anatomy of the superficial veins of the cerebrum. Neurosurgery 1985;17:711-48 CrossRef Medline

10. Ueyama $\mathrm{T}, \mathrm{Al}-\mathrm{Mefty} \mathrm{O}$, Tamaki $\mathrm{N}$. Bridging veins on the tentorial surface of the cerebellum: a microsurgical anatomic study and operative considerations. Neurosurgery 1998;43:1137-45 CrossRef Medline

11. Miabi Z, Midia R, Rohrer SE, et al. Delineation of lateral tentorial sinus with contrast-enhanced MR imaging and its surgical implications. AJNR Am J Neuroradiol 2004;25:1181-88 Medline

12. Vatansever A, Mut M, Ergun KM, et al. The anatomy of the sigmoid-transverse junction according to the tentorial angle. $J$ Craniofac Surg 2019;30:2280-84 CrossRef Medline

13. Browder K, Kaplan HA, Krieger AJ. Venous channels in the tentorium cerebelli: surgical significance. Surg Neurol 1975;3:37-39 Medline

14. Browder J, Kaplan HA, Krieger AJ. Anatomical features of the straight sinus and its tributaries: clinical correlations. J Neurosurg 1976;44:55-61 CrossRef Medline
15. Kaplan HA, Browder J, Krieger AJ. Venous channels within the intracranial dural partitions. Radiology 1975;115:641-45 CrossRef Medline

16. Chung JI, Weon YC. Anatomic variations of the deep cerebral veins, tributaries of basal vein of Rosenthal: embryologic aspects of the regressed embryonic tentorial sinus. Interv Neuroradiol 2005;11:123-30 CrossRef Medline

17. Rosenblum JS, Neto M, Essayed WI, et al. Tentorial venous anatomy: cadaveric and radiographic study with discussion of origin and surgical significance. World Neurosurg 2019;131:e38-45 CrossRef Medline

18. Padget DH. The cranial venous system in man in reference to development, adult configuration, and relation to arteries. Am J Anat 1956;98:307-55 CrossRef Medline

19. Streeter GL. The developmental alterations in the vascular system of the brain of the human embryo. In: Contributions to Embryology Carnegie Institution No. 24. Carnegie Institution of Washington; 1921

20. Velut S. Embryology of the cerebral veins [in French]. Neurochirurgie 1987;33:258-63 Medline

21. Schulter FP. A comparative study of the temporal bone in three populations of man. Am J Phys Anthropol 1976;44:453-68 CrossRef Medline

22. Lee DH, Kim MJ, Lee $\mathrm{S}$, et al. Anatomical factors influencing pneumatization of the petrous apex. Clin Exp Otorhinolaryngol 2015; 8:339-44 CrossRef Medline

23. Kao SC, Waziri MH, Smith WL, et al. MR imaging of the craniovertebral junction, cranium, and brain in children with achondroplasia. AJR Am J Roentgenol 1989;153:565-69 CrossRef Medline

24. Cramér H. Mathematical Methods of Statistics. Princeton University Press; 1946:282

25. Hastie T, Tibshirani R, Friedman J. Hierarchical clustering. In: Hastie T, Tibshirani R, Friedman J, eds. The Elements of Statistical Learning, 2nd ed. Springer-Verlag 2009:520-28

26. Hierarchical Clustering. https://docs.scipy.org/doc/scipy/reference/ cluster.hierarchy.html\#module-scipy.cluster.hierarchy. Accessed November 3, 2019

27. Rokach L, Maimon O. Clustering methods. In: Maimon O, Rokach L, eds. Data Mining and Knowledge Discovery Handbook. SpringerVerlag; 2005:321-52

28. scipy.spatial.distance.pdist. https://docs.scipy.org/doc/scipy/reference/ generated/scipy.spatial.distance.pdist.html. Accessed November 3, 2019

29. Press WH, Flannery BP, Teukolsky SA, et al. Information-theoretic properties of distributions. In: Press WH, Teukolsky SA, Vetterling WT, et al, eds. Numeric Recipes: The Art of Scientific Computing. 3rd ed. Cambridge University Press; 1992

30. Okudera T, Huang YP, Ohta T, et al. Development of the posterior fossa dural sinuses, emissary veins, and jugular bulb: morphological and radiologic study. AJNR Am J Neuroradiol 1994;15:1871-83 Medline

31. Mitsuhashi Y, Hayasak K, Kawakami T, et al. Dural venous system in the cavernous sinus: a literature review and embryological, functional, and endovascular clinical considerations. Neurol Med Chir (Tokyo) 2016;56:326-39 CrossRef Medline 